Franz J. Wegner

\title{
In memory of Leo P. Kadanoff
}

Received: Accepted:

\begin{abstract}
Leo Kadanoff has worked in many fields of statistical mechanics. His contributions had an enormous impact. This holds in particular for critical phenomena, where he explained Widom's homogeneity laws by means of block-spin transformations and laid the basis for Wilson's renormalization group equation. I had the pleasure to work in his group for one year. A short historically account is given.
\end{abstract}

Keywords Renormalization group · Block-spin transformation · Critical phenomena $\cdot$ Ising Model $\cdot$ Duality

\section{Introduction}

Leo Kadanoff has worked in many fields of Statistical Mechanics.

He started out in working on superconductivity in a thesis under the supervision of Paul Martin at Harvard. Leo and Gordon Baym developed self-consistent approximations which preserved the conservation laws[4]. They published the widely used book Conservation laws and correlation functions [34]. Gordon Baym has reviewed his time with Leo in his contribution Conservation laws and the quantum theory of transport: The early days.[3]

After a number of papers related to superconductivity and transport phenomena he became interested in critical phenomena, where he contributed essentially. Section 2 reviews shortly the situation in this field in the fifties and sixties of the last century. Section 3 reviews the contributions of Ben Widom and Leo Kadanoff in 1965 and 1966, which were two important steps in the understanding of this field. This led to a strongly growing interest in this field (sect. 4). Finally, in 1971 Ken Wilson developed the tool to calculate explicitly the critical behavior. In the same year Rodney Baxter solved the eight-vertex model and enriched the class of two-dimensional exactly solvable critical models. (sect. 5) I had the great pleasure

Institute for Theoretical Physics, Ruprecht-Karls-University, Heidelberg 
to be in Leo Kadanoff's group in this year. A very short account of Leo's work in the following years, which is mainly in critical phenomena, is given in section 6 .

\section{Critical phenomena}

In the fifties and sixties of the last century a lot of investigations started to understand the behavior of gas-liquid systems, magnets and other systems close to their critical point.

The theory by Van der Waals[55] for gas-liquid systems and that by Curie and Weiss [65] for magnetic substances predicted what is called molecular field behavior. In terms of the paramagnetic-ferromagnetic transition one describes the critical behavior by

$$
m \propto(-\tau)^{\beta}, \quad \chi \propto|\tau|^{-\gamma}, \quad c \propto|\tau|^{-\alpha}, \quad \xi \propto|\tau|^{-v}
$$

at zero magnetic field, where $m$ is the magnetization, $\chi$ the susceptibility, $c$ the specific heat, $\xi$ the correlation length, and $\tau=\left(T-T_{c}\right) / T_{c}$ measures the difference of the temperature $T$ to its critical value $T_{c}$. For liquid-gas systems $m$ has to be replaced by the difference $\rho-\rho_{c}$ of the density and its critical value. The exponent $\alpha=0$ corresponds to a jump and/or a logarithmic divergence of the specific heat. Molecular field approximation yields a jump in the specific heat and critical exponents $\beta=1 / 2, \gamma=1, v=1 / 2$.

Landau[44] formulated a general theory, which enclosed not only gas-liquid systems and magnetic materials, but many others like binary mixtures and the transition to superfluid helium. He described the systems in terms of an order parameter which for magnetic systems is the magnetization and in gas-liquid systems is the difference of the density and the critical density.

Experimentally one found $\beta \approx 1 / 3, \gamma \approx 4 / 3$, and values of $\alpha$ between -0.1 and +0.1 . Part of these observations date back to 1900 . See the review by LeveltSengers. [47]

Two exact solutions showed that the critical behavior might differ from molecular field behavior: The three-dimensional spherical[5] model yields $\gamma=2$ and a kink in the specific heat, but no jump, corresponding to $\alpha=-1$; the twodimensional Ising model yields $\beta=1 / 8[72]$ and a logarithmically diverging specific heat [50]. Moreover, Ginzburg[11] analyzed the fluctuation contributions near the critical point and came to the conclusion that in three dimensions there is a region where molecular field theory fails, its temperature-range depending on the range of interaction.

A way to obtain the critical exponents was to perform expansions for lattice models like the Ising model and classical Heisenberg models in powers of the coupling over temperature. From these expansions one could estimate the critical temperature and critical exponents. They came close to experimentally determined exponents. Unfortunately however, one did not understand the mechanism behind this behavior. 


\section{Widom 1965 and Kadanoff 1966}

There were three important steps to understand critical behavior: The first step was Ben Widom's homogeneity law[66] in 1965, according to which the order parameter is a homogeneous functions of $\tau$ and a second quantity, which for the gas-liquid transition is the difference $\mu-\mu_{c}$ of the chemical potential and its critical value. For magnets one uses instead the magnetic field $h$. This was an extremely useful concept. It explained the relations between critical exponents, which were already known as equalities or inequalities. Several experiments were analyzed accordingly and good agreement was found. Examples are the measurements of the magnetization of $\mathrm{CrBr}_{3}$ by Ho and Litster[16] and of nickel by Kouvel and Comly [42], and the equation of state for various gases by Green, VicentiniMissoni, and Levelt-Sengers[12].

In 1966 Leo Kadanoff[21] presented the second important step to understand critical phenomena with his block transformation. He replaced the spins within a block by a new block spin and introduced an effective interaction between the block spins, which yields the same behavior for magnetization and spin correlations on distances large in comparison to the block size. Denote the original coupling and magnetic field by $K$ and $h$ and the block one by $K^{\prime}$ and $h^{\prime}$. The number of spins within the block be $b^{d}$. Then there is a mapping of $(K, h) \rightarrow\left(K^{\prime}, h^{\prime}\right)$ : $K^{\prime}=f(K)$, and the magnetic field will effectively change by a factor one may call $b^{x}: h^{\prime}=b^{x} h$. It is essential that this transformation is not singular, at least not close to the critical coupling $K_{c}$. This critical coupling reproduces itself $K_{c}=f\left(K_{c}\right)$ under the block transformation. Small deviations from $K_{c}$, which one may call $\tau=K-K_{c}$ increase under the block transformation by a factor we may call $b^{y}$, $\tau^{\prime}=b^{y} \tau$. Then the two exponents $x$ and $y$ determine the critical exponents

$$
\alpha=2-d / y, \quad \beta=(d-x) / y, \quad \gamma=(2 x-d) / y, \quad v=1 / y .
$$

\section{Interest grows}

In 1967 the interest in critical phenomena had strongly grown. Several reviews appeared on this subject: the reviews by Michael Fisher[10], Peter Heller[15], and Leo Kadanoff[36] and his coworkers.

In 1970 I participated in the Midwinter Solid State Research Conference on the topic Critical Phenomena. Among the participants were many prominent workers in this field. I mention only Günter Ahlers, George Baker, Richard Ferrell, Michael Fisher, Robert Griffiths, Bertrand Halperin, Peter Heller, Pierre Hohenberg, Leo Kadanoff, David Landau, J.D. Litster, Paul Martin, Michael Schulhoff, Johanna Levelt-Sengers, Eugene Stanley, Gerard Toulouse, Ken Wilson, Michael Wortis, and Peter Young. It was my first travel to the United States and it was a pleasure to meet many physicists in person, which before I knew only from their papers. It was also interesting for me, since I had applied for a post-doc position with several of them.

Laramore [45] wrote a short report on this conference and concerning static critical phenomena he resumed: The two-parameter Kadanoff-Widom scaling laws are in real trouble as far as predicting the relationships between the static critical exponents in three-dimensional systems, the deviation from the scaling laws being 
small, but nevertheless real. Thus at that time there were doubts on this theory. One should be aware that the effect of dipolar interactions and of anisotropies including crossover effects were not yet sufficiently clear. However, dipolar effects in uniaxial ferroelectrics were already considered by Larkin and Khmelnitskii[46], and crossover effects by Jasnow and Wortis[18] and by Riedel and myself[51].

\section{Baxter and Wilson 1971}

1971 saw two very important advances in critical phenomena, the solution of the eight-vertex model by Rodney Baxter and the renormalization group calculation of Ken Wilson on the basis of Kadanoff's block spin ideas.

In 1971 I was research-associate in Leo Kadanoff's group. It turned out that at that time Leo was engaged in urban planning, but was still interested in critical phenomena. When I came to Brown University I had already started with thinking about: How can the idea of duality as introduced by Kramers and Wannier[43] for the two-dimensional Ising model be generalized to higher dimensions? Kadanoff and Ceva[35] had introduced the concept of the disorder variable, which under duality transforms into the Ising spin. This concept was useful for my considerations. I realized that the dual model to the conventional three-dimensional Ising model is an Ising model with plaquette interactions. In four dimensions the Ising model with plaquette interactions is self-dual.[56] Thus its transition temperature, provided there is only one, can be determined in the same way and with the same result as for the conventional two-dimensional Ising model. The transition temperature for this model was confirmed later numerically by Creutz, Jacobs, and Rebbi $[8]$ to the precision allowed by the hysteresis effects at the first-order transition. The model has a local gauge invariance. The products of spins along loops, called Wilson-loops [69], show different behavior in the two phases. They decay with increasing loops with either an area law or a perimeter law, depending on the phase

$$
\left\langle\prod_{\text {loop }} S(r)\right\rangle \propto \begin{aligned}
& \exp (-a / a(T)) \\
& \exp (-p / p(T))
\end{aligned},
$$

where $a$ is the area, and $p$ the perimeter of the loop. What I did not realize at that time, was that this behavior is characteristic for the behavior of confinement and deconfinement of quarks.

1971 saw the solution of the eight-vertex model by Rodney Baxter[1,2]. The model is equivalent to an Ising model on a square lattice with two-spin interactions and a four-spin interaction of strength $\lambda$. If $\lambda$ vanishes, then the system decays into two conventional Ising models. Starting from this limit Leo and myself determined the critical exponents in order $\lambda$. [41] This is only possible, since the system has an operator, which stays marginal, and creates a line of fixed-points with varying exponents.

Later during that year the papers by Wilson 67,68$]$ appeared, in which he derived an approximate renormalization group equation, which allowed him to determine critical exponents for the three-dimensional Ising model. This was the third important step to understand critical phenomena. These papers were based on Kadanoff's block spin picture. 
Wilson stated that the space of Hamiltonians is much larger than the one used by Kadanoff, but Kadanoff has used all relevant operators necessary to reach the critical point and has thus obtained the correct picture in the immediate vicinity of the critical point. Doubts on the Widom-Kadanoff picture soon declined.

Starting from this picture the concept of a fixed point and of universality classes became clear. Thus all systems whose interactions converge at criticality to the same fixed point, are governed by the same critical exponents and - as later became clear - also to the same amplitude ratios. I investigated aspects of the general structure of the flow of the interaction under renormalization, considering also irrelevant operators and that the renormalization includes non-linear flows of the couplings[57]. What assured me that Wilson's approach is the correct approach was the paper by Wilson and Fisher[70] on the $\varepsilon$-expansion in $d=4-\varepsilon$ dimensions. A theory, which in complete agreement with the Ginzburg[11] criterion gave molecular field behavior for $d>4$ and exponents different from molecular field behavior for $d<4$ has the expected properties. Wilson's approximate recursion relation was so good that it gave the correct critical exponents in order $\varepsilon$. We (Grover, Kadanoff and FJW) used it to determine the exponents for the threedimensional classical Heisenberg model[13]. I added a calculation of the critical exponents for the isotropic $n$-vector model[58]. It happened several times that I did not understand an answer by Leo immediately, but only one or two days later. But his answers were so concise that I remembered them after such a long time.

It is surprising that Ken Wilson developed first this approximate renormalization group equation and only later came back to the Landau free-energy functional. The free theory contains only a two-spin interaction $\sum_{q} u(q) S_{q} S_{-q}$ with (i)

$u(q) \propto q^{2}$ for small wave-vectors $q$, and (ii) $u(q) \rightarrow 1$ for $q$ large in comparison to the cut-off momentum $\Lambda$. The first condition is obvious and can be easily incorporated in the transformation, but the formulation of the transformation for the second condition is less obvious. It can be found in refs. [71] and [59].

I became also known to Anthony Houghton at Brown University. A different way which Anthony Houghton, and myself[64] used, was a sharp momentum cut-off. This procedure works well in first order in $\varepsilon$, but yields potentials nonanalytic in the wave-vector for higher orders in $\varepsilon$. We also obtained the behavior of the n-component vector model in the limit $n \rightarrow \infty$. It agreed with the spherical model[5] in accordance with Stanley [54]. Our paper became known as functional renormalization, since we calculated the interaction as a function.

\section{Later Years}

After my stay at Brown I met Leo only occasionally at conferences. Once he visited Heidelberg for a talk.

Obviously Leo wanted to learn all secrets of two-dimensional and if possible three-dimensional critical systems. This includes correlation functions[22,24] as well as operator algebras [23, 26] and renormalization group procedures in real space, which are characterized by potential moving and variational schemes 25 , 37, 38, 53,9]. He studied extensively the renormalization scheme[27] by Migdal[48, 49]. With Jorge Jose, Scott Kirkpatrick, and David Nelson he investigated the ef- 
fect of vortices and symmetry-breaking anisotropic fields on the planar model at low temperatures [20,28].

But he studied also other systems showing critical behavior as turbulence[6], avalanches [39]7], and the critical behavior of Kolmogorov-Arnold-Moser surfaces [40, 29,30], and mappings, which create critical boundaries.

Some work of Leo[14,19] together with Halsey, Jensen, Procaccia, and Shraiman on fractal measures came close to some work of our work on the Anderson transition in a random potential. At the mobility edge, which separates extended and localized eigenstates, the states approach a fractal behavior. In a block picture one diagonalizes first the interaction within small blocks. This is continued with larger and larger blocks. This yields a transformation in real space and energy space.[60]. After the mapping of this Anderson model to the nonlinear sigmamodel was found [61,52] it was possible to determine the fractal exponents of the eigenfunctions in $2+\varepsilon$ dimensions [62, 17,63].

Leo characterized his work at several occasions [31, 32, 33].

Unfortunately I missed Leo, when he was in Chicago. I spent a sabattical at the James-Franck Institute in the summer of 1978, whereas Leo moved from Brown University to the James-Frank Institute in the winter of 1978. I am glad that I met him in 2015 at the March meeting of the American Physical Society in San Antonio. He was still intensively interested in the progress of Physics.

\section{References}

1. R. Baxter, Eight-Vertex Model in Lattice Statistics, Phys. Rev. Lett. 26,832 (1971)

2. R. Baxter, Partition function of the eight-vertex lattice model, Annals of Phys. 70, 193 (1972)

3. G. Baym, Conservation Laws and the quantum theory of transport: The early days, http://jifi.uchicago.edu/ leop/Kb.pdf

4. G. Baym and L.P. Kadanoff, Conservation Laws and Correlation Functions, Phys. Rev. 124, $237(1961)$

5. T.H. Berlin and M. Kac, The spherical model of a ferromagnet, Phys. Rev. 86, 821 (1952)

6. B. Castaign, G. Gunaratne, F. Heslot, L.P. Kadanoff, A. Libchaber, S. Thomae, X.-Z. Wu, S. Zalewski, and G. Zanetti, Scaling of Hard Turbulence in Rayleigh Benard Convection, J. Fluid Mech. 209, 1 (1989)

7. A.B. Chhabra, M.L. Feigenbaum, L.P. Kadanoff, A.J. Kolan, and I. Procaccia, Sandpiles, Avalanches, and the statistical mechanics of nonequilibrium stationary states, Phys. Rev. B47, 3099 (1993)

8. M. Creutz, L. Jacobs, and C. Rebbi, Experiments with a gauge-invariant Ising system, Phys. Rev. Lett. 42, 1390 (1979)

9. E. Efrati, Z. Wang, A. Kolan, and L.P.Kadanoff, Real-space renormalization in statistical mechanics, Rev. Mod. Phys. 86, 647 (2014)

10. M.E. Fisher, The theory of critical phenomena, Rep. Prog. Phys. 30, 615 (1967)

11. V.L. Ginzburg, Some remarks on phase transitions of the second kind and the microscopic theory of ferroelectric materials, Fiz. Tverd. Tela, 2, 2031 (1960); Sov. Phys. Solid State 2, $1824(1960)$

12. M.S. Green, M. Vicentini-Missoni, and J.M.H. Levelt-Sengers, Scaling-Law Equation of State for Gases in ther Critical Region, Phys. Rev. Lett. 18, 1113 (1967)

13. M.K. Grover, L.P. Kadanoff, and F.J. Wegner, Critical Exponents for the Heisenberg Model, Phys. Rev. B6, 311 (1972)

14. T.C. Halsey, M.H. Jensen, L.P. Kadanoff, I. Procaccia, and B.I. Shraiman, Fractal Measures and their Singularities: the Characterization of Strange Sets, Phys. Rev. A 33, 1141 (1986); Erratum Phys. Rev. A 34, 1601 E (1986)

15. P. Heller, Experimental investigations of critical phenomena, Rep. Prog. Phys. 30, 731 (1967) 
16. J.T. Ho and J.D. Litster, Magnetic equation of state of $\mathrm{CrBr}_{3}$ near the critical point, Phys. Rev. Lett. 22, 603 (1969)

17. D. Höf, F. Wegner, calculation of anomalous dimensions for the nonlinear sigma model, Nucl. Phys. B275, 561 (1986)

18. D. Jasnow and M. Wortis, High temperature critical indices for the classical anisotropic Heisenberg model, Phys. Rev. 176, 739 (1968)

19. M.H. Jensen, L.P. Kadanoff, and I. Procaccia, Scaling Structure and Thermodynamics of Strange Sets, Phys. Rev. A36, 1409 (1987)

20. J.V. Jose, L.P. Kadanoff, S. Kirkpatrick, and D.R. Nelson, Renormalization, Vortices, and symmetry-breaking perturbations in the two-dimensional planar model, Phys. Rev. B16, 1217 (1977)

21. L.P. Kadanoff, Scaling laws for Ising models near $T_{c}$, Physics 2, 263 (1966)

22. L.P. Kadanoff, Spin-Spin Correlations in the Two-dimensional Ising model, Nuovo Cim. 44 $276(1966)$

23. L.P. Kadanoff, Operator Algebra and the Determination of Critical Indices, Phys. Rev. Lett. 23, 1430 (1969)

24. L.P. Kadanoff, Correlations along a Line in the Two-Dimensional Ising Model, Phys. Rev. 188,859 (1969)

25. L.P. Kadanoff, Variational Principles and Approximate Renormalization Group Calculations, Phys. Rev. Lett. 34, 1005 (1975)

26. L. P. Kadanoff, Scaling, Universality, and Operator Algebras in Phase Transitions and Critical Phenomena ed. C. Domb and M.S. Green, Vol. 5A (New York, Academic Press), (1976) p.1

27. L.P. Kadanoff, Notes on Migdal's Recursion Formulas, Ann. Phys. 100, 359 (1976)

28. L.P. Kadanoff, Multicritical Behavior at the Kosterlitz-Thouless Critical Point, Ann. Phys. 120, 39 (1979)

29. L.P. kadanoff, Scaling for a Critical Kolmogorov-Arnold-Moser (KAM) Trajectory, Phys. Rev. Lett. 47, 1641 (1981)

30. L.P. Kadanoff, Critical behavior of a KAM Surface: II. Renormalization Approach, in Melting, Localization, and Chaos, ed. R.K. Kalia and P. Vashista, North Holland, New York (1982)

31. L.P. Kadanoff, Scaling and Universality in Statistical Physics, Physica A163, 1 (1990)

32. L.P. Kadanoff, From Order to Chaos, World Scientific (1993)

33. L. P. Kadanoff, Interview in the Physics of Scales project, (2002) http://authors.library.caltech.edu/5456/1/hrst.mit.edu/hrs/renormalization/Kadanoff/index.htm

34. L.P. Kadanoff and G. Baym, Quantum Statistical Mechanics (W. A. Benjamin, New York) (1962)

35. L.P. Kadanoff and H. Ceva, Determination of an Operator Algebra for the Two-Dimensional Ising Model, Phys. Rev. B3, 3918 (1971)

36. L.P. Kadanoff, W. Götze, D. Hamblen, R. Hecht, E.A.S. Lewis, V.V. Palciauskas, M. Rayl, J. Swift, D. Aspnes, and J. Kane, Static phenomena near critical points: Theory and experiment, Rev. Mod. Phys. 39, 395 (1967)

37. L.P. Kadanoff and A. Houghton, Numerical Evaluations of the Critical Properties of the Two-Dimensional Ising Model, Phys. Rev. B11, 377 (1975)

38. L.P. Kadanoff, A. Houghton, and M.C. Yalabik, Variational Approximations for Renormalization Group Transformations J. Stat. Phys. 14, 171 (1976)

39. L.P. Kadanoff, S.R. Nagel, L. Wu, and S-M. Zhoum Scaling and Universality in Avalanches, Phys. Rev. A39, 6524 (1989)

40. L.P. Kadanoff and S.J. Shenker, Critical Behavior of a KAM Surface: I. Empirical Results, J. Stat. Phys. 27, 631 (1982)

41. L.P. Kadanoff and F.J. Wegner, Some critical properties of the eight-vertex model, Phys. Rev. B4, 3989 (1971)

42. J.S. Kouvel and J.B. Comly, Magnetic equation of state for Nickel near its Curie point, Phys. Rev. Lett. 20, 1237 (1968)

43. H.A. Kramers and G.H. Wannier, Statistics of the two-dimensional ferromagnet. Part I, Phys. Rev. 60, 252 (1941)

44. L.D. Landau, On the theory of Phase Transitions, Zh. Eksp. Teor. Fiz. 7, 19 (1937); reprinted in Collected Papers, (Nauka, Moscow, 1969) Vol. 1, 234

45. G.E. Laramore, A report on the midwinter solid-state research conference, J. Stat. Phys. 2, $107(1970)$ 
46. A.I. Larkin and D.E. Khmelniskii, Phase transitions in Uniaxial Ferroelectrics, Zh. Eksp, Teor, Fiz, 56, 2087 (1969); JETP 29, 1123 (1969)

47. J.M.H. Levelt-Sengers, From Van der Waals' equation to the scaling laws, Physica 73, 73 (1974)

48. A.A. Migdal, Recursion equation in gauge field theories Zh. Eksp. Teor. Fiz. 69, 810 (1975); Sov. Phys. JETP 42, 413 (1976)

49. A.A. Migdal, Phase transitions in gauge and spin-lattice systems Zh. Eksp. Teor. Fiz. 69 , 1457 (1975); Sov. Phys. JETP 42, 743 (1976)

50. L. Onsager, Crystal statistics. I. A two-dimensional model with an order-disorder transition, Phys. Rev. 65, 117 (1944)

51. E. Riedel and F. Wegner, Scaling approach to anisotropic magnetic systems, statics, Z. Physik 225, 195 (1969)

52. L. Schäfer and F. Wegner, Disordered system with $n$ orbitals per lattice site: Lagrange formulation, hyperbolic symmetry, and Goldstone modes, Z. Phys. B38, 113 (1980)

53. S.J. Shenker, L.P. Kadanoff, and A. Pruisken, A variational Real Space Renormalization Group Transformation based on the Cumulant Expansion, J. Phys. A12, 91 (1979)

54. H.E. Stanley, Spherical model as the limit of infinite spin dimensionalily, Phys. Rev. 176, $718(1968)$

55. J.D. van der Waals, On the continuity of the gas and liquid state, thesis (in Dutch), Leiden (1873)

56. F.J. Wegner, Duality in generalized Ising models and phase transitions without local order parameter, J. Math. Phys. 12, 2259 (1971)

57. F.J. Wegner, Corrections to scaling laws, Phys. Rev. B5, 4529 (1972)

58. F.J. Wegner, Critical exponents in isotropic spin systems, Phys. Rev. B6, 1891 (1972)

59. F.J. Wegner, The critical state, general aspects in in Phase Transitions and Critical Phenomena, eds. C. Domb and M. Green, Vol. 6, (Academic Press, New York), p.7 (1976)

60. F.J. Wegner, Electrons in Disordered Systems. Scaling near the Mobility Edge, Z. Phys. B25, 327 (1976)

61. F. Wegner, The mobility edge problem: Continuous symmetry and a conjecture, Z. Phys. B35, 207 (1979)

62. F. Wegner, Inverse Participation Ratio in $2+\varepsilon$ dimensions, Z. Phys. B36, 209 (1980)

63. F. Wegner, Anomalous Dimensions for the nonlinear Sigma-Model in $2+\varepsilon$ dimensions, Nucl. Phys. B280, 210 (1987)

64. F.J. Wegner and A. Houghton, Feynman-graph calculation of the $(0, l)$ critical exponents to order $\varepsilon^{2}$, Phys. Rev. A10, 435 (1974)

65. P. Weiss, L'hypothese du champ moléculaire et la propriété ferromagnétique, J. Phys. Theor. Appl. 6, 661 (1907)

66. B. Widom, Equation of State in the neighborhood of the critical point, J. Chem. Phys 43, $3898(1965)$

67. K.G. Wilson, Renormalization group and critical phenomena. I. Renormalization group and the Kadanoff scaling picture, Phys. Rev. B4, 3174 (1971)

68. K.G. Wilson, Renormalization group and critical phenomena. II. Phase-space cell analysis of critical behavior, Phys. Rev. B4, 3184 (1971)

69. K.G. Wilson, Confinement of quarks, Phys. Rev. D10, 2445 (1974)

70. K.G. Wilson and M.E. Fisher, Critical exponents in 3.99 dimensions, Phys. Rev. Lett. 28, $240(1972)$

71. K.G. Wilson and J. Kogut, The renormalization group and the $\varepsilon$ expansion, Phys. Rep. 12C, 75 (1974)

72. C.N. Yang, The spontaneous magnetization of a two-dimensional Ising model, Phys. Rev. $85,808(1952)$ 